\title{
Comparison of Ensemble and Single Molecule Methods for Particle Characterization and Binding Analysis of a PEGylated Single Domain Antibody
}

\author{
Linda J. Obenauer-Kutner ${ }^{1}$ and Lumelle A. Schneeweis ${ }^{2}$ \\ 1. Parenteral Science and Technology, Bristol-Myers Squibb, New Brunswick, New Jersey. \\ 2. Protein Science and Structure, Bristol-Myers Squibb, Princeton, New Jersey.
}

Domain antibodies ( $\mathrm{dAb}$ ) are single immunoglobulin domains that form the smallest functional unit of an antibody. This study investigated the behavior of these small proteins when covalently attached to the polyethylene glycol (PEG) moiety that is necessary for extending the half-life of this small protein. The effect of the 40kDa PEG on hydrodynamic properties, particle behavior, and receptor binding of the dAb has been compared by both ensemble solution methods and single molecule atomic force microscopy methods. AFM offered a unique strength for characterizing protein-protein interactions and complemented many of the techniques used routinely in the Biotech and Pharmaceutical industries. SEC-MALS, dynamic light scattering (DLS) and AFM topography measurements provided orthogonal methods to characterize the hydrodynamic radius and particle properties of the dAb-1-PEG conjugate in comparison to the non-PEGylated dAb-1. Though the PEG is conjugated at a site distant from the paratope, given that the PEG is four times the size of the $\mathrm{dAb}-1$ protein, the PEG moiety has the potential to change the functional characteristics of dAb-1 such as its binding kinetics and affinity, and its fractional activity. AFM recognition imaging efficiency (Figure 1) and unbinding force curves (Figure 2) provided an orthogonal method to SPR and ITC solution ensemble methods that measure the single molecule binding interaction of the dAb-1-PEG conjugate with its target antigen. Ultimately, the comparison of ensemble methods (SEC-MALS, SPR, ITC) with AFM single molecule topography and recognition imaging methods provided an improved understanding of the dAb-1-PEG conjugate particle properties and how the PEG impacts the interaction of dAb-1with the target antigen.

Overall, the use of single molecule methods for particle characterization and receptor binding characterization offers the ability to investigate particle structure and heterogeneity that complements the mass, kinetics, and affinity measured by ensemble [1]. PEGylation of protein therapeutic molecules is a common method for prolonging serum half-life. In the case of small, single domain protein therapeutics such as domain antibodies, the PEG contribute significantly to the particle characteristics and hydrodynamic properties with very minimal impact on the thermodynamics of the protein-protein interaction with its target antigen. 


\section{References:}

[1] L Schneeweis, et al, Journal of Pharmaceutical Sciences (2015) p. 4015

Dr. S. Lindsay and Dr. P Kaur are thanked for their many useful discussions and contributions to this work.

Figure 1. Atomic force microscopy recognition image (topography \& comparable recognition). Dimensions of images shown are 1000 x $1000 \mathrm{~nm}$.

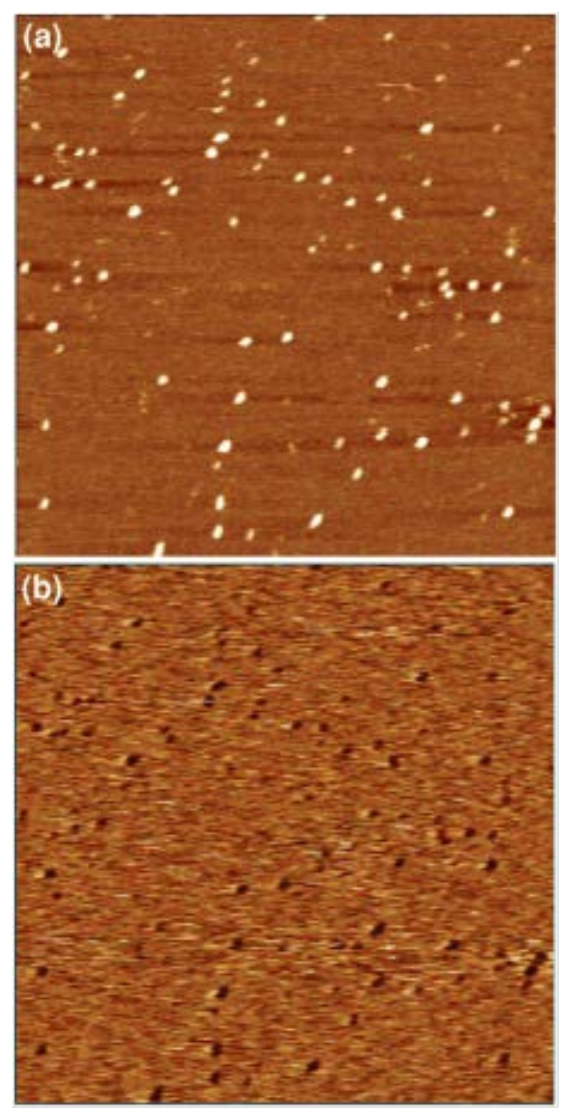

Figure 2. Histograms of AFM frequency versus unbinding force.

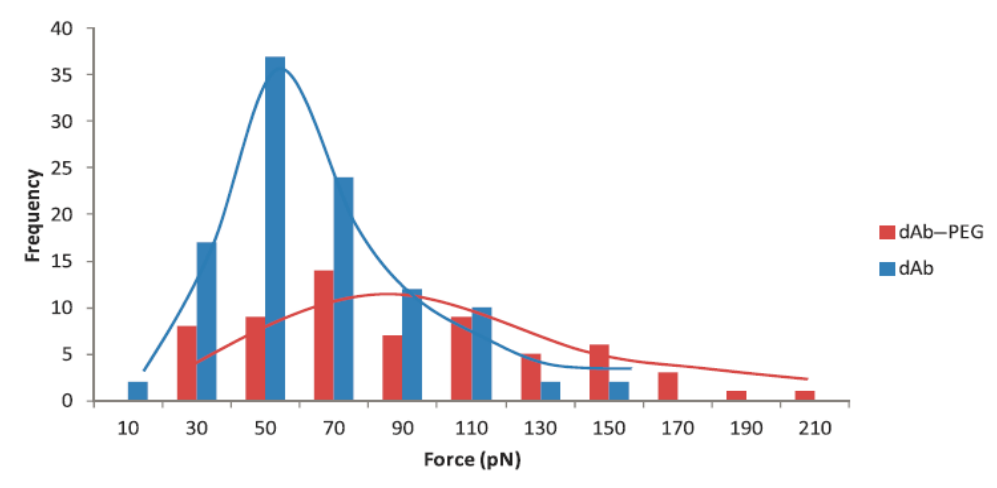

